\title{
THERMAL INFRARED CAMERA FOR MOBILE DEVICES
}

\author{
Youngrae $\mathrm{Jo}^{1 \dagger}$, Bongki Back ${ }^{1}$, Byungjun Yeo ${ }^{1}$ and Sanggu Kang ${ }^{1 *}$ \\ ${ }^{1}$ I3system, Inc., Gajeongbuk-ro 26-32, Yuseong-gu, Daejeon 34113, Republic of Korea \\ †Presenting Author: jyr@i3system.com \\ "Corresponding Author: kangsg@i3system.com
}

\begin{abstract}
The infrared camera is useful for night vision, detecting fire, measuring temperatures and detecting target in smoke. The price of the infrared cameras is rapidly decreased. Those reasons explain why infrared camera market is expanded and needs the infrared camera with small size, low weight and low power consumption (SWaP) for portability. Thermal Expert ${ }^{\mathrm{TM}}$ with low SwaP and highest resolution is developed by I3system as the business needs. The image of Thermal Expert is displayed with the smartphone. In this paper, Thermal Expert is presented with the performance compared with the infrared cameras of other suppliers in the world.
\end{abstract}

KEYWORDS: Infrared Camera, Micro Bolometer, I3System, Thermal Expert, Mobile device

\section{INTRODUCTION}

In the beginning, the infrared camera is mainly used for military services. Recently, the application area of the infrared camera is growing. The infrared camera can also be used for non-destructive test. For example, when the defect of the electric component or the power line of the telephone is occurred, heat is generated more than usual. The infrared camera can detect which component or the power line is defective. Also, the infrared camera is used to detect if there are problems for the adiabatic condition of the building as nondestructive test.

The business market for the infrared camera with portability and usability is growing. And the demand for the uncooled infrared camera is growing than the cooled infrared camera. Moreover, the thermoelectric cooler(TEC) and shutter for image calibration[1] is removed in the uncooled infrared camera for low SwaP applications. Developing the infrared camera with low SWaP is the one of the main topic in the research field of infrared camera.

The display device for the image of infrared camera is usually not portable. Because of this, the smart phone is used as a substitute for the display device recently. In order to meet the market requirements, I3system has developed Thermal Expert displayed with a smartphone. Other companies have developed the similar infrared cameras, FLIR one[2], Seek-Thermal[3] and Therm-app[4], to Thermal Expert.

\section{THERMAL EXPERT}

I3system has developed the infrared camera Thermal Expert that is low SWaP and a device that the image is displayed with a smartphone. In this chapter, the concept, functions, actual applications and specification of Thermal Expert are presented. There are details of Thermal Expert on http://www.i3-thermalexpert.com.

\subsection{CONCEPT}

An appearance of Thermal Expert Q1 is presented in Fig. 2. The dimension is $47 \mathrm{~mm} \times 25 \mathrm{~mm} \times 16 \mathrm{~mm}$ and the weight is less than $39 \mathrm{~g}$ with lens. This means Thermal Expert is compact and portable. And the smartphone is used as a substitute for the display device. There are two software to display the image of 
Thermal Expert. One is the android application and the other is the windows application. Because it is difficult to analyze the details of images on the smartphone, a user can analyze the images saved by a smartphone with the analysis program on the computer(Fig. 2).
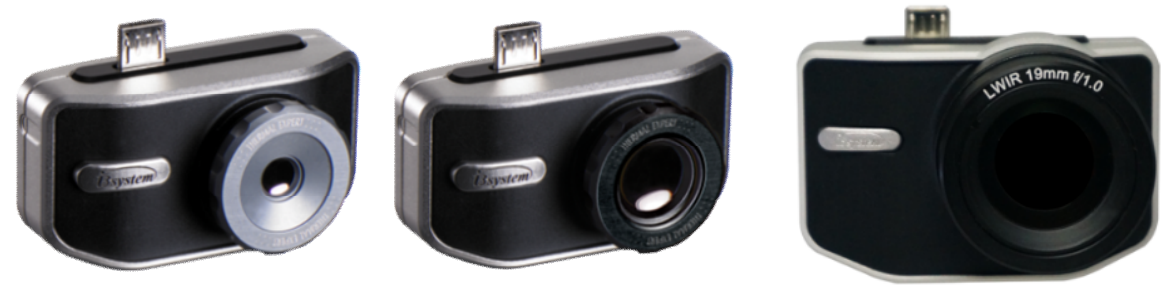

Fig. 1 Thermal Expert. The two models, Thermal Expert Q1 with lens $6.8 \mathrm{~mm}$ and $13 \mathrm{~mm}$ (option) on the left. Thermal Expert V1 on the right.

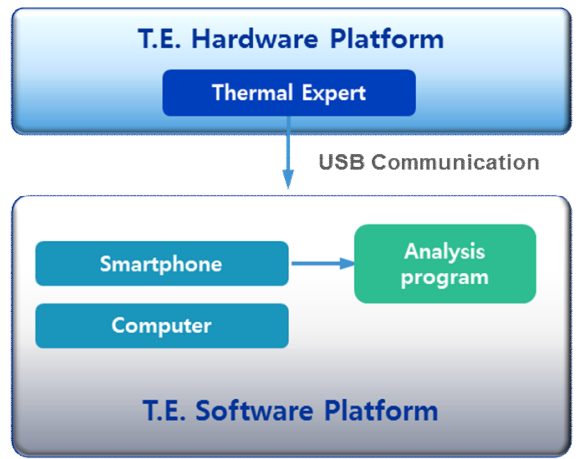

Fig. 2 Block diagram for the concept of Thermal Expert.

\subsection{FUNCTIONS}

In this paper, some important functions is just presented. First, it measures the temperature. In the app, the user can measure the temperature with points, rectangles, lines and circles. Second, there are the alarm function. In the rectangle, if the temperature of the target is over the reference temperature, the alarm sounds and the target is colored red to alert(Fig. 3(a)). Third, the visible image and the infrared image can be display both on the screen of the smartphone(Fig. 3(b)). The user can control the contrast and the brightness with the level span bar. The image saved with Thermal Expert can be analyzed in detail on the compute. A user can make the simple report on the android application for Thermal Expert and the detail report with the analysis program of Thermal Expert. And the report indicates the location where the image is saved by using GPS signal served on the smartphone(Fig. 3(c)). And a user can use Thermal Expert on Windows OS of the computer. These two software tools are provided on the website of Thermal Expert.

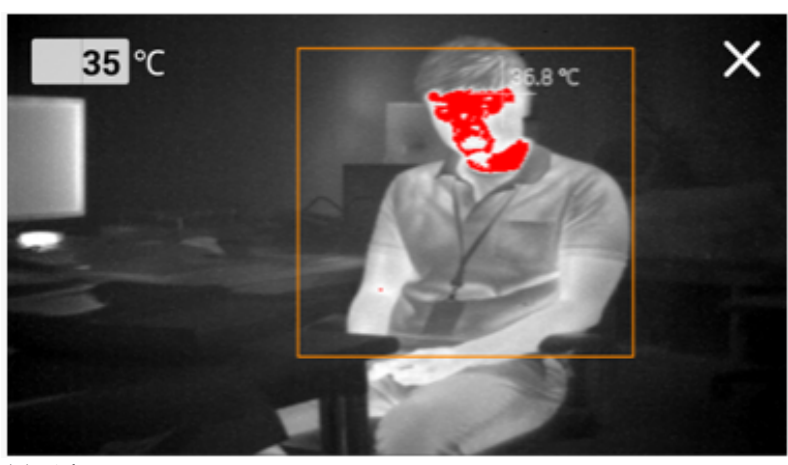

(a) Alarm

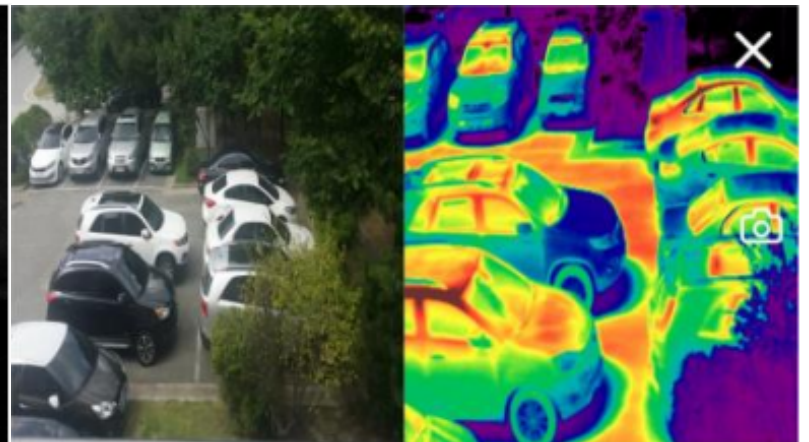

(b) Both the visible image and the infrared image display 


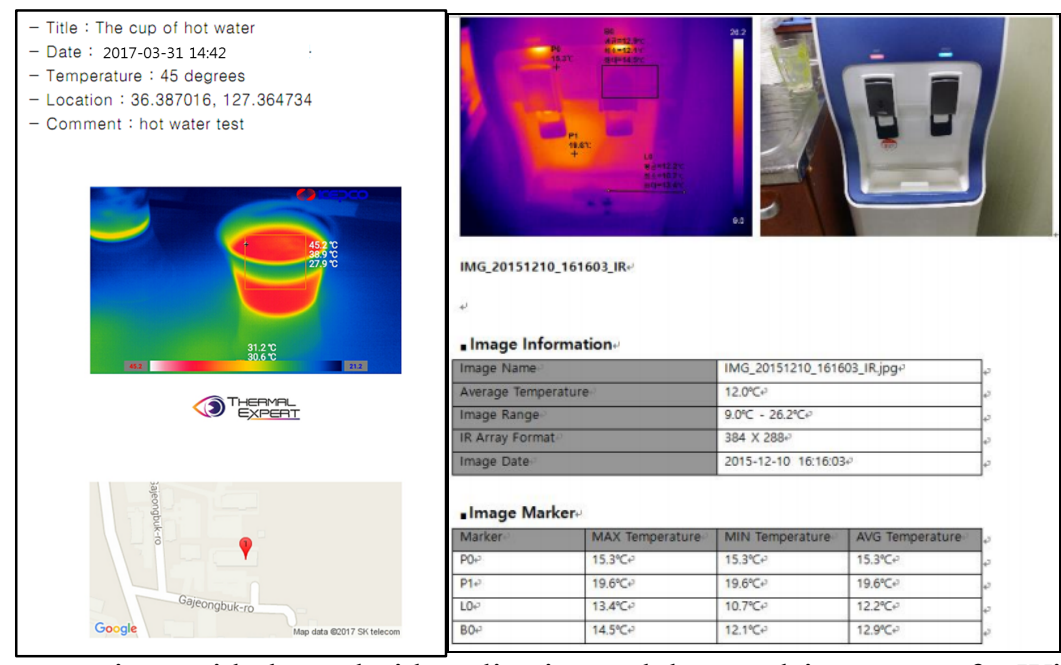

(c) Report written with the android application and the anaylsis program for Windows

Fig. 3 The functions of Thermal Expert.

\subsection{APPLICATIONS}

In this chapter, the example for using Thermal Expert for the non-destructive test is presented. First, the repairman for the power transmission and distribution device and the utility power pole wants to know which device is going to be destructive before it is really destructive(Fig. 3(a)). The temperature of electric components that have the problem is higher than usual and the repairman can know if it has the problem. Second, the developer can know which electric component is the reason why the power consumption is high in the electric board(Fig. 3(b)). Third, Thermal Expert can be used to test heat wire within the electric pad before it is sell(Fig. 3(c)).

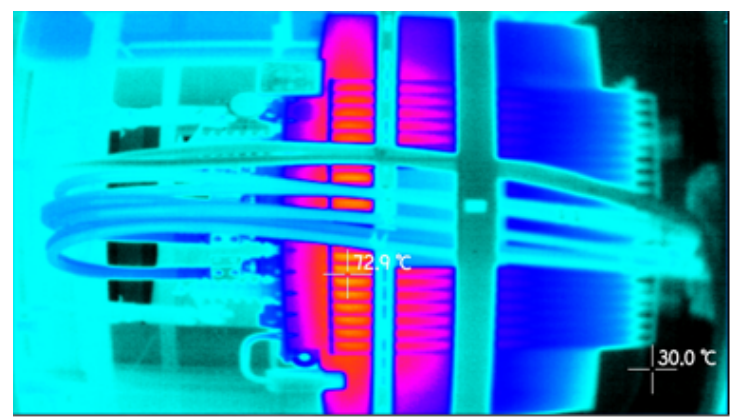

(a) Power transmission and distribution system test

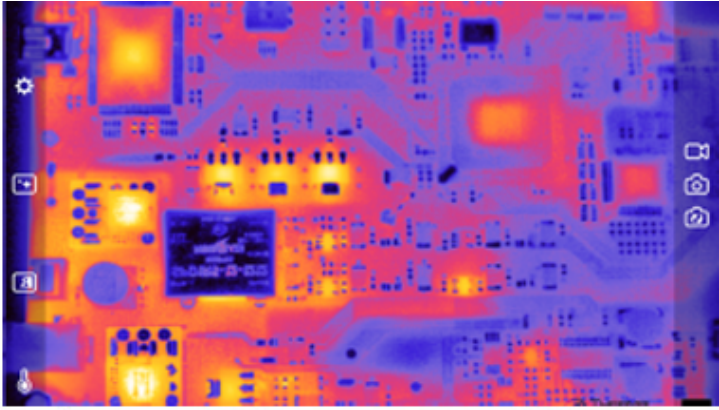

(b) Power consumption test of the PCB board

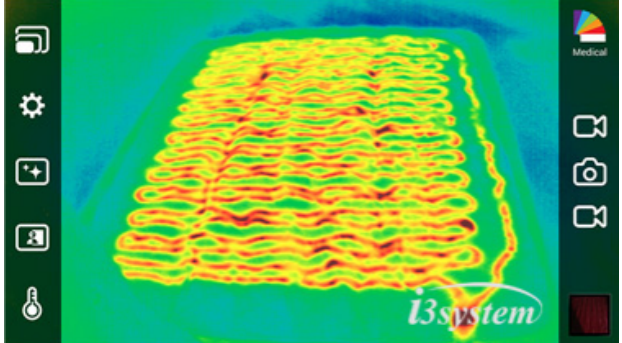

(c) Heat wire of the electric pad test

Fig. 4 The application for non-destructive test with Thermal Expert. 


\subsection{SPECIFICATION}

In table 1, the specification of Thermal Expert Q1, V1, Therm-App, Seek Thermal, FLIR One(Gen2) is described. The resolution of Thermal Expert is 384x288. This resolution is higher than the infrared camera of other companies. But the resolution of Therm-App is same with the resolution of Thermal Expert. But the image of Therm-App is not so good due to lower NETD of the detector used. The sensitivity of Thermal Expert is high. Thermal Expert shows excellent thermography operation at comparably affordable price versus industrial thermography cameras.

Table 1 Specification.

\begin{tabular}{|c|c|c|c|c|c|}
\hline Spec. & $\begin{array}{c}\text { Thermal } \\
\text { Expert Q1 }\end{array}$ & $\begin{array}{l}\text { Thermal } \\
\text { Expert V1 }\end{array}$ & Therm-App & Seek Thermal & $\begin{array}{c}\text { FLIR } \\
\text { One(Gen2) } \\
\end{array}$ \\
\hline Resolution & $\begin{array}{c}384 \times 288 \\
(110 \mathrm{~K})\end{array}$ & $\begin{array}{c}640 \times 480 \\
(300 \mathrm{~K})\end{array}$ & $\begin{array}{c}384 \times 288 \\
(110 \mathrm{~K})\end{array}$ & $\begin{array}{c}206 \times 156 \\
(32 \mathrm{~K})\end{array}$ & $\begin{array}{c}160 \times 120 \\
(19 \mathrm{~K})\end{array}$ \\
\hline Pixel Pitch $[\mu \mathrm{m}]$ & 17 & 17 & 17 & 12 & 17 \\
\hline $\begin{array}{r}\text { Weight [g] } \\
\text { (with lens) }\end{array}$ & 27 & 90 & 138 & $20 \sim 42$ & 78 \\
\hline $\begin{array}{l}\text { Dimensions }[\mathrm{mm}] \\
\text { (without lens) }\end{array}$ & $47 \times 25 \times 16$ & $50 \times 32 \times 30$ & $55 \times 65 \times 40$ & $40 \times 20 \times 15$ & $72 \times 26 \times 18$ \\
\hline Sensitivity[mK] & 50 & 50 & 70 & - & 100 \\
\hline Operation Temp. $\left[{ }^{\circ} \mathrm{C}\right]$ & $-10 \sim 50$ & $-20 \sim 50$ & $-10 \sim 50$ & - & $0 \sim 35$ \\
\hline Scene range Temp. $\left[{ }^{\circ} \mathrm{C}\right]$ & $-10 \sim 250$ & $-10 \sim 120$ & $5 \sim 90$ & $-40 \sim 330$ & $-20 \sim 120$ \\
\hline Product Image & & & & & \\
\hline
\end{tabular}
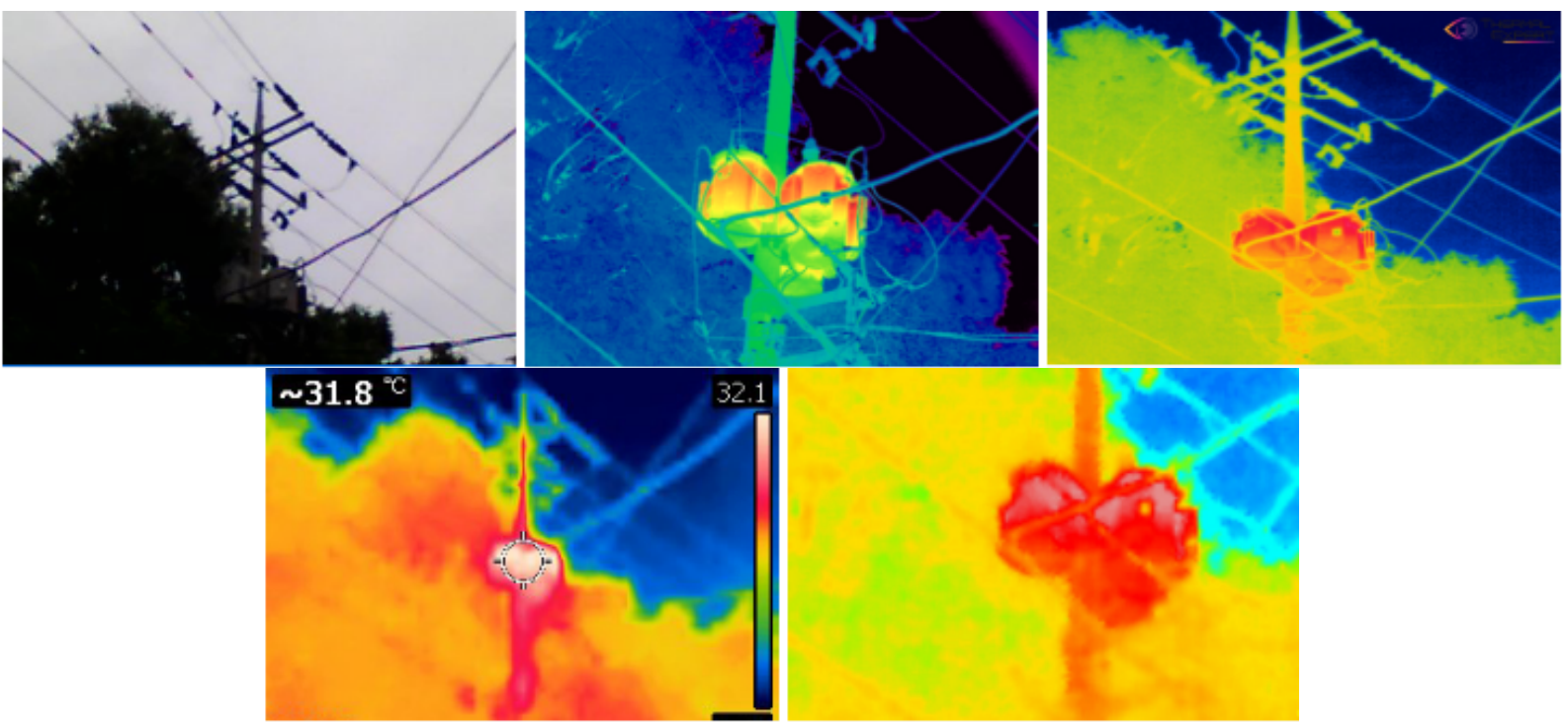

(a) The image of the telephone pole(the visible image, Thermal Expert V1, Thermal Epxert Q1, FLIR C2[5], Seek Thermal from left to right)

Fig. 5 The image comparison of infrared cameras for mobile device. 


\section{CONCLUSIONS}

Thermal Expert is used for the many applications of non-destructive. The presented infrared camera, Thermal Expert, shows promising results comparing with other similar infrared cameras. In the next study, the image processing apply to Thermal Expert to improve the image quality of Thermal Expert.

\section{REFERENCES}

[1] A. Tempelhahn, H. Budzier, V. Krause, G. Gerlach, Shutter-less calibration of uncooled infrared cameras., in: J. Sens. Sens. Syst., 2016: pp. 9-16.

[2] http://www.flir.com/flirone/ios-android/.

[3] http://www.thermal.com/.

[4] https://therm-app.com/.

[5] http://www.flir.com/instruments/c2/ 